Int. J. Electrochem. Sci., 14 (2019) 10259 - 10269

\title{
Crystal Structure, Surface Activity and Corrosion Inhibition Effect of Dihydroxyethyl Ammonium O,O'-di(4-methylpheny) dithiophosphate for Q235 Steel in $1.0 \mathrm{M} \mathrm{H}_{2} \mathrm{SO}_{4}$
}

Chuan Lai ${ }^{1,2,3 *}$, Jing Cao ${ }^{2}$, Yuan-fang Deng ${ }^{2}$, Ya-fei Yang ${ }^{2}$, Xin Wen ${ }^{2}$, Zhu-li Wang ${ }^{2}$, Cheng-wu Fan ${ }^{1}$, Ya-nan Shi ${ }^{1}$, Ying Li ${ }^{1}$, Jun-lan Li ${ }^{1}$, Chang-lin Yang ${ }^{1}$, Yi-jiu Yang ${ }^{1}$, Wei Pang ${ }^{1}$, Yang Liu ${ }^{1}$

${ }^{1}$ School of Chemistry and Chemical Engineering, Sichuan University of Arts and Science, Dazhou 635000, China

${ }^{2}$ DaZhou Quality Technical Supervision and Inspection Testing Center, Dazhou 635000, China

${ }^{3}$ Laboratories of Fine Chemicals and Surfactants in Sichuan Provincial Universities, Sichuan University of Science and Engineering, Zigong, 643000, China

*E-mail: laichuanemail@163.com

doi: $10.20964 / 2019.11 .27$

Received: 16 June 2019 / Accepted: 10 August 2019 / Published: 7 October 2019

The hydrophilic corrosion inhibitor of dihydroxyethyl ammonium O,O'-di(4-methyl pheny)dithiophosphate (DAOP) was synthesized. Meanwhile, the crystal structure, surface activity and corrosion inhibition of DAOP were presented in this work. The weight loss and potentiodynamic polarization measurements results all shows that DAOP is an effective hydrophilic corrosion inhibitor for Q235 steel (QS-235) in $1.0 \mathrm{M} \mathrm{H}_{2} \mathrm{SO}_{4}$. Potentiodynamic polarization measurements present that DAOP is a mixed-type inhibitor, which adsorbed on QS-235 surface attributable to both physisorption and chemisorption.

Keywords: Synthesis; Acid; Surface activity; Inhibitor; Chemisorption.

\section{FULL TEXT}

(C) 2019 The Authors. Published by ESG (www.electrochemsci.org). This article is an open access article distributed under the terms and conditions of the Creative Commons Attribution license (http://creativecommons.org/licenses/by/4.0/). 